\title{
ERRATUM
}

\section{Erratum to: Machine learning identification of symmetrized base states of Rydberg atoms}

\author{
Daryl Ryan Chong ${ }^{1}$, Minhyuk Kim² ${ }^{2}$ Jaewook Ahn ${ }^{2}$, Heejeong Jeong ${ }^{1, \dagger}$ \\ ${ }^{1}$ Department of Physics, Faculty of Science, University of Malaya, Kuala Lumpur 50603, Malaysia \\ ${ }^{2}$ Department of Physics, KAIST, Daejeon 34141, Korea \\ Corresponding author. E-mail: ${ }^{\dagger} j$ hj413@gmail.com
}

ERRATUM TO: Front. Phys. 17(1), 12504 (2022),
https://doi.org/10.1007/s11467-021-
1099-0.

The online version of the original article can be found at https://doi.org/10.1007/s11467-021-1099-0 and http:// journal.hep.com.cn/fop/EN/10.1007/s11467-021-1099-0.

The $x$-axis of Fig. 3(b) should be labelled "Observation duration" instead of "Total evolution time" as shown below.
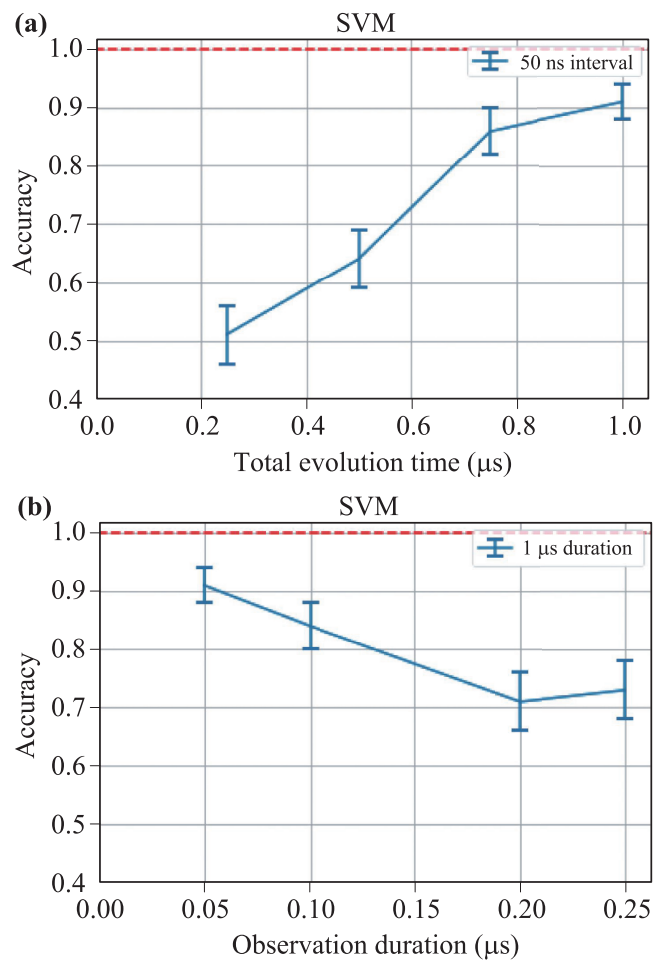

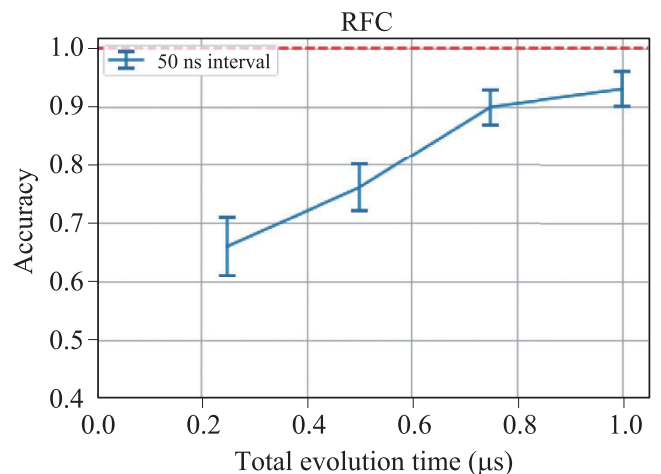

RFC

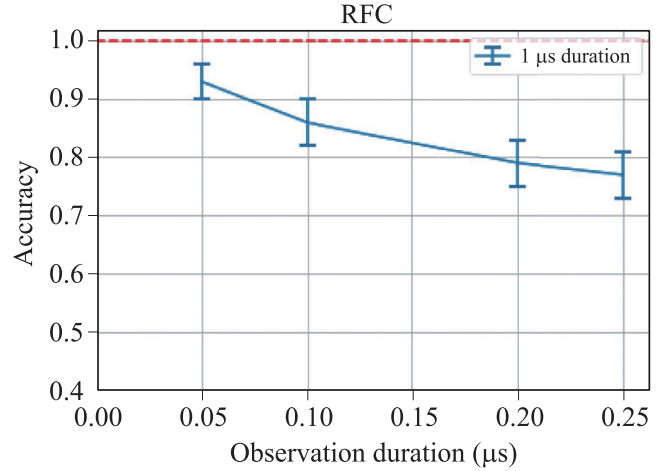

Fig. 3 Accuracy performance depending on (a) total evolution time, and (b) observation duration between data points for the case of identification of $\mathrm{Na}$ in Figs. 2(a, c, e). The accuracy values are obtained by utilizing both SVM and RFC. The default total evolution time is $1 \mu \mathrm{s}$, and the default duration is $50 \mathrm{~ns}$ for all our ML sample data in this paper. The results show our default experimental parameters provides above $90 \%$ accuracy as in Figs. 2(a, c, e).

\footnotetext{
*Special Topic: Trapped Atoms and Ions for Quantum Science (Eds. Le Luo, Kenji Toyoda, Kihwan Kim, Jaewook Ahn \& Dzmitry Matsukevich).
} 